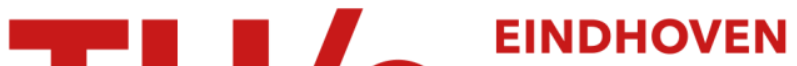 UNIVERSITY OF TECHNOLOGY
}

\section{Theoretical investigation of a photoconductively switched high- voltage spark gap}

Citation for published version (APA):

Broks, B. H. P., Hendriks, J., Brok, W. J. M., Brussaard, G. J. H., \& Mullen, van der, J. J. A. M. (2006). Theoretical investigation of a photoconductively switched high-voltage spark gap. Journal of Applied Physics, 99(12), 123302-1/9. [123302]. https://doi.org/10.1063/1.2204756

DOI:

$10.1063 / 1.2204756$

Document status and date:

Published: 01/01/2006

Document Version:

Publisher's PDF, also known as Version of Record (includes final page, issue and volume numbers)

Please check the document version of this publication:

- A submitted manuscript is the version of the article upon submission and before peer-review. There can be important differences between the submitted version and the official published version of record. People interested in the research are advised to contact the author for the final version of the publication, or visit the $\mathrm{DOI}$ to the publisher's website.

- The final author version and the galley proof are versions of the publication after peer review.

- The final published version features the final layout of the paper including the volume, issue and page numbers.

Link to publication

\section{General rights}

Copyright and moral rights for the publications made accessible in the public portal are retained by the authors and/or other copyright owners and it is a condition of accessing publications that users recognise and abide by the legal requirements associated with these rights.

- Users may download and print one copy of any publication from the public portal for the purpose of private study or research.

- You may not further distribute the material or use it for any profit-making activity or commercial gain

- You may freely distribute the URL identifying the publication in the public portal.

If the publication is distributed under the terms of Article 25fa of the Dutch Copyright Act, indicated by the "Taverne" license above, please follow below link for the End User Agreement:

www.tue.nl/taverne

Take down policy

If you believe that this document breaches copyright please contact us at:

openaccess@tue.nl

providing details and we will investigate your claim. 


\title{
Theoretical investigation of a photoconductively switched high-voltage spark gap
}

\author{
B. H. P. Broks, J. Hendriks, W. J. M. Brok, G. J. H. Brussaard, and \\ J. J. A. M. van der Mullen ${ }^{\mathrm{a}}$ \\ Department of Applied Physics, Eindhoven University of Technology, P.O. Box 513, 5600 MD Eindhoven, \\ The Netherlands
}

(Received 28 November 2005; accepted 13 April 2006; published online 22 June 2006)

\begin{abstract}
In this contribution, a photoconductively switched high-voltage spark gap with an emphasis on the switching behavior is modeled. It is known experimentally that not all of the voltage that is present at the input of the spark gap is switched, but rather a fraction of it drops across the spark gap. This voltage drop depends on the voltage that is present at the input of the spark gap with higher voltages resulting in a smaller drop. We have investigated two possible causes of this: the cathode fall and the resistance of the plasma arc. Using an analytical model of the cathode fall, we have established that the cathode fall can be excluded as the cause of the observed voltage drop. A one-dimensional, time-dependent non-local thermal equilibrium fluid model of the arc plasma has been made. Using this model, the plasma properties have been analyzed for various values of the switched current with emphasis on the conductivity. A good qualitative match between the observed and the simulated dissipation in the gap was found. This indicates that the finite arc resistance is the cause of the observed voltage drop. (C) 2006 American Institute of Physics. [DOI: 10.1063/1.2204756]
\end{abstract}

\section{INTRODUCTION}

Spark gaps are used for the rapid switching of high voltages and high currents in a wide range of applications. Our interest in spark gaps lies mainly in the construction of compact pulsed dc electron accelerators. ${ }^{1}$ With these accelerators, electric fields can be achieved that are an order of magnitude higher than the electric fields that can be achieved with conventional radio frequency accelerators. For these compact dc accelerators, high-voltage pulses with a short rise time, short duration, and favorably no time jitter are required.

Spark gaps have been used to switch large voltages for over a century. A conventional spark gap consists of two electrodes with a gas-filled gap between them. When a voltage higher than the breakdown voltage is applied across the spark gap, breakdown occurs, and the gas between the electrodes ionizes. An arc plasma with a high degree of ionization and good conductivity forms, closing the spark gap.

The breakdown process occurs by Townsend avalanches and streamers and requires that the ambient electron density of, for instance, air, which is typically $10^{9} \mathrm{~m}^{-3}$, increases by several orders of magnitude to achieve good conduction. This is an inherently stochastic process, lengthening the rise time and producing time jitter.

By axially focusing a laser into the spark gap, the initial ionization degree of air can be raised by several orders of magnitude, creating a well-defined preionized channel between the electrodes. ${ }^{2}$ The higher initial electron density smoothes statistical fluctuations, and the electron density has to rise less to achieve good conductivity. Because of these

${ }^{a)}$ Electronic mail: j.j.a.m.v.d.mullen@tue.nl two effects, the rise time and jitter are shorter, as low as subnanosecond, while allowing pulses in the order of $1-100 \mathrm{kV}$ to be transmitted. ${ }^{3}$

The use of Ti:sapphire lasers that can produce terawatt pulses with a duration in the femtosecond range allows the concept of the laser-triggered spark gap to be taken one step further. By focusing the laser between the electrodes, a plasma can be created with an ionization degree that is so high that no further ionization is necessary to achieve good conduction: in other words, the laser not so much triggers the spark gap, but rather switches it. Recently, we have reported on experiments, ${ }^{4,5}$ in which a terawatt laser was used to photoconductively switch voltages of up to $4.5 \mathrm{kV}$ with a jitter better than the resolution of the measuring equipment, $12 \mathrm{ps}$. Furthermore, this switch is capable of switching voltages as low as $10 \%$ of the self-breakdown voltage.

However, in these experiments it was observed that, in particular, for lower voltages, a significant part of the voltage drops across the spark gap due to plasma processes. This voltage drop can be observed in Fig. 1, where the input voltage $V_{\text {in }}$ and the output voltage $V_{\text {out }}$ are plotted.

In this contribution, we will investigate in detail two probable causes of the observed voltage drop, namely, the cathode fall and the plasma arc resistance, to determine the cause of the voltage drop over the discharge using theoretical models. In Sec. II, a description of the setup will be given. In Sec. III a simple, analytical model of the cathode fall formation in the laser-produced plasma has been made, and based on this, the relevance of the cathode fall to the switching behavior will be discussed. In Sec. IV, a time-dependent numerical model of the arc plasma in the spark gap is described. Using this model, an analysis of the plasma and the 


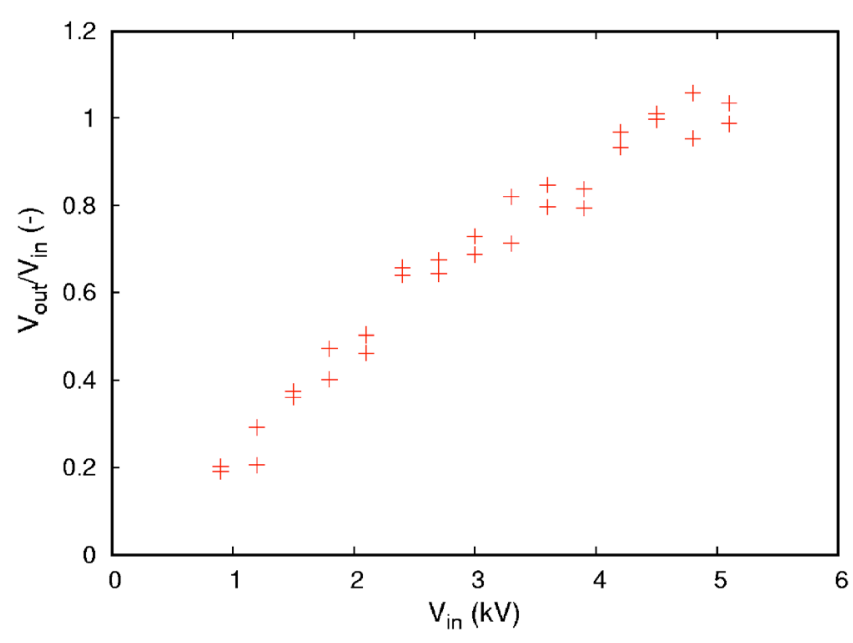

FIG. 1. (Color online) The fraction of the voltage that is switched by the spark gap as a function of the input voltage as presented in Ref. 5. For high voltages, almost all the voltage is switched, and only a small fraction of the voltage is lost across the spark gap. With decreasing voltage, an increasing fraction of the voltage is lost across the spark gap. The input voltage is limited to $5 \mathrm{kV}$ because of self break down.

switching behavior is made that is much more detailed than the estimate of the plasma resistance made in Ref. 5.

\section{THE SETUP}

The experimental setup has been described in detail in Refs. 4 and 5. In this section, the discussion will be limited to the most essential parts.

The spark gap is schematically drawn in Fig. 2. The spark gap is basically a $1 \mathrm{~mm}$ interruption in a coaxial transmission line structure that has a characteristic impedance of $55 \Omega$. One side of this structure is charged to a high voltage $V_{\text {in }}$, in our case $4.5 \mathrm{kV}$, limited by the self-breakdown voltage of the spark gap. The gap is filled with either nitrogen or air at atmospheric pressure. Because there is little experimental difference between the switching behaviors of the gases, ${ }^{5}$ the discussion will be restricted to the case of nitrogen.

The gap is switched using a Ti:sapphire laser that has a pulse energy of $35 \mathrm{~mJ}$ and a pulse duration of $200 \mathrm{fs}$. The laser is focused into a filament that connects the two electrodes to cylindrical lenses. The intensity over the entire gap is above the intensity threshold for tunnel ionization. The focus has a full width at half maximum (FWHM) height of

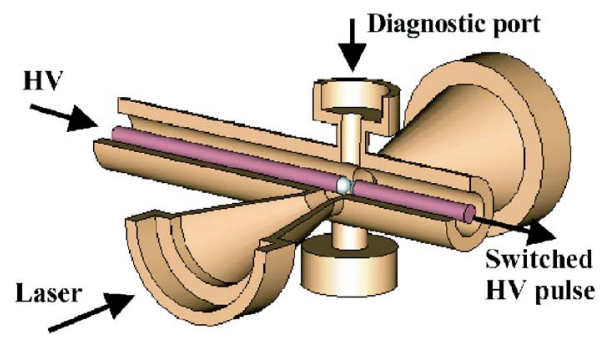

FIG. 2. (Color online) A schematic drawing of the spark gap switch adapted from Refs. 4 and 5. The gap is an interruption in a coaxial transmission line structure and is filled with either nitrogen or air. By focusing a terawatt laser in a line focus in the spark gap, a laser-produced plasma bridges the gap between the electrodes, switching the voltage that is over the gap.
$20 \pm 3 \mu \mathrm{m}$, a length of $949 \pm 6 \mu \mathrm{m},{ }^{5}$ and a width of approximately $100 \pm 3 \mu \mathrm{m}$. The radius of curvature of the electrodes is $3 \mathrm{~mm}$. This means that the plasma is a thin filament that connects both electrodes, while it fills only a fraction of the gap between the electrodes. The switched pulse has a length of $2 \mathrm{~ns}$ and a rise time of less than $100 \mathrm{ps}$.

As mentioned in Sec. I, we will model two different aspects of the plasma: the initial cathode fall formation and the evolution of the arc plasma between the electrodes. The initial plasma composition and size have not been measured and have been estimated from the size of the laser focus and the power density in the focus. A schematic representation of both model areas is given in Fig. 3.

For the modeling of the cathode fall plasma, an analytical model is used, which requires the size of the plasma as an input. The plasma is approximated by a homogeneous slab, with a size determined by the FWHM of the laser focus: $l$ $=1 \mathrm{~mm}, w=100 \mu \mathrm{m}$, and $h=20 \mu \mathrm{m}$. We will see in Sec. III that this model is of a sufficient accuracy to discuss the importance of the cathode fall.

For the modeling of the arc plasma, a one-dimensional, time-dependent model is used. The plasma is considered to be homogeneous in the $x$ and $y$ directions, with $l=1 \mathrm{~mm}$ and $w=100 \mu \mathrm{m}$ while the $z$ direction, in which the largest gradients appear, is resolved on a numerical grid.

\section{THE CATHODE FALL}

To conduct current, a spark gap plasma requires a mechanism that supplies electrons from the cathode to the plasma. In a conventional arc, the electrodes are hot, and thermionic emission is the main source of electrons from the cathode. In this case, however, the cathode is initially cold and does not heat up significantly in the short discharge time $(\sim 2 \mathrm{~ns})$. Hence, thermionic emission cannot produce a significant amount of electrons; instead, field emission and secondary emission must be the dominant processes supplying electrons to the discharge. Both these processes are enhanced by a cathode fall, a region of positive space charge in front of the cathode. This creates an electric field that may liberate electrons directly due to field emission and accelerates ions toward the cathode, causing secondary emission.

In this section, we will present a simple, analytical model of the plasma, which we use to describe the formation of the cathode fall. We are particularly interested in an upper estimate of the voltage drop over the cathode fall $V_{\text {cath }}$, which acts as a parasitic loss in the switch, and in an upper estimate of the time needed to form a cathode fall $t_{\text {cath }}$, as this may limit the switching time.

In this analytical model, we will approximate the laserproduced plasma by a uniform slab, with dimensions as shown in Fig. 3 and an $n_{e}$ of $5 \times 10^{23} \mathrm{~m}^{-3}$. The density estimate is based on an analysis of the laser intensity, and is detailed in Sec. IV B, and is equivalent to an ionization degree of about $5 \%$. The mechanism of the cathode fall formation in this system will be explained using the graphs in Fig. 4.

The initial plasma is produced by the femtosecond laser pulse at a time scale that is much shorter than the plasma 


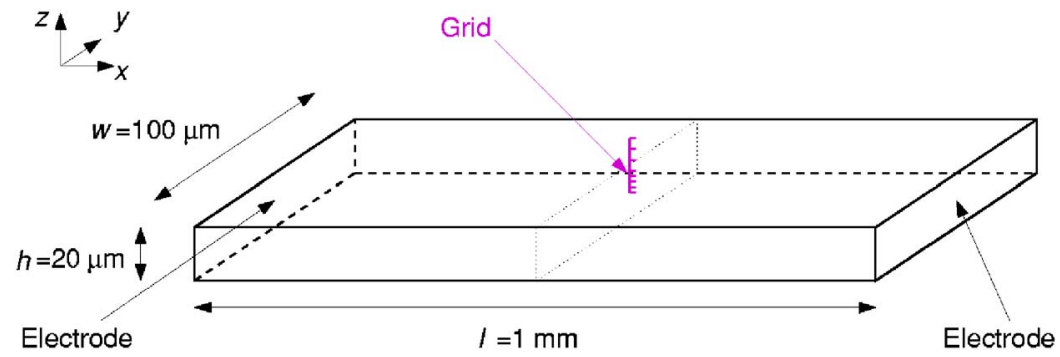

FIG. 3. (Color online) Schematic representation of model approximation of the laser-produced plasma. The plasma is assumed to be uniform in the $x$ and $y$ directions. In the cathode fall model of Sec. III, the plasma is also assumed to be uniform in the $z$ direction. In the arc model of Sec. IV, the plasma properties vary along $z$; the grid in which the numerical calculation of the plasma properties is performed is shown, superimposed on a cross section of the plasma. Symmetry is used, and only the top half of the plasma is simulated. On the top left, the coordinate system, as used throughout this work, is drawn. The size of the slab is based on measurements detailed in Ref. 5. The radius of curvature of the electrodes is $3 \mathrm{~mm}$, hence, they can be considered as planar on the length scale of the plasma.

processes under consideration. The top graph of Fig. 4 schematically shows the laser-produced plasma density and the applied voltage. In the initial laser-produced plasma, the electric field applied across the spark gap will move electrons to the anode. The ion movement to the cathode is orders of magnitudes slower and negligible at this time scale. The movement of the electrons leaves a region near the cathode depleted of electrons, with a positive space charge that is very high due to the high ion density. The very high positive charge density causes an electric field to form near the cathode that is much higher than the average field, which facilitates the electron emission. This process is shown in the bottom graph of Fig. 4.

\section{A. The cathode fall voltage}

We will now estimate an upper bound of the cathode fall voltage. For this, the electron emission current from the cathode as a function of the electric field at the cathode $E_{\text {cath }}$ has been calculated using the Fowler-Nordheim equation ${ }^{6,7}$

$$
I_{\text {field }}=K_{4} E_{\text {cath }}^{2} A \exp \left(\frac{-K_{5}}{E_{\text {cath }}}\right),
$$

in which $K_{4}$ and $K_{5}$ are emission coefficients and $A$ is the cathode area. As noted in Ref. 6, (1) typically overestimates the field required for significant field emission by at least one order of magnitude. This is due to surface roughness, which may greatly enhance the local field and hence the emission. A typical value for this field enhancement parameter $\beta$ is 50 , and we will use this value in the calculation.

Incorporating $\beta$ in (1) and substituting values for $K_{4}$ and $K_{5}$ we obtain: ${ }^{8}$

$$
\begin{aligned}
I_{\text {field }}= & \frac{1.54 \times 10^{-6} \times 10^{4.52 \phi^{-0.5}}\left(\beta E_{\text {cath }}\right)^{2} A}{\phi} \\
& \times \exp \left(\frac{-6.53 \times 10^{9} \phi^{1.5}}{\beta E_{\text {cath }}}\right),
\end{aligned}
$$

with $E_{\text {cath }}$ in $\mathrm{V} \mathrm{m}^{-1}$, the work function $\phi$ in $\mathrm{eV}, A$ in $\mathrm{m}^{2}$, and $I_{\text {field }}$ in A.

The cathode tips consist of a tungsten-copper alloy, which has an unknown work function. The work function of tungsten $\phi_{\mathrm{W}}$ is $4.54 \mathrm{eV}$ (Ref. 9) and the work function of copper $\phi_{\mathrm{Cu}}$ is $4.65 \mathrm{eV} .{ }^{10,11}$ The values of the work function of an alloy is typically between the value of the work function of the constituents. ${ }^{12}$

For an electrode area of $2 \times 10^{-9} \mathrm{~m}^{2}$ (cf. Fig. 3), $I_{\text {field }}$ as a function of $E_{\text {cath }}$ has been plotted in Fig. 5 for both tungsten and copper cathodes. Figure 5 shows that $I_{\text {field }}$ is negligible for values of $E_{\text {cath }}$ lower than $9 \times 10^{7} \mathrm{~V} \mathrm{~m}^{-1}$ and increases very rapidly with increasing $E_{\text {cath }}$ for higher values of $E_{\text {cath }}$. The maximum switched current of $50 \mathrm{~A}$ can be supplied by field emission by a field of $1.2 \times 10^{8} \mathrm{~V} \mathrm{~m}^{-1}$. Noting that con-
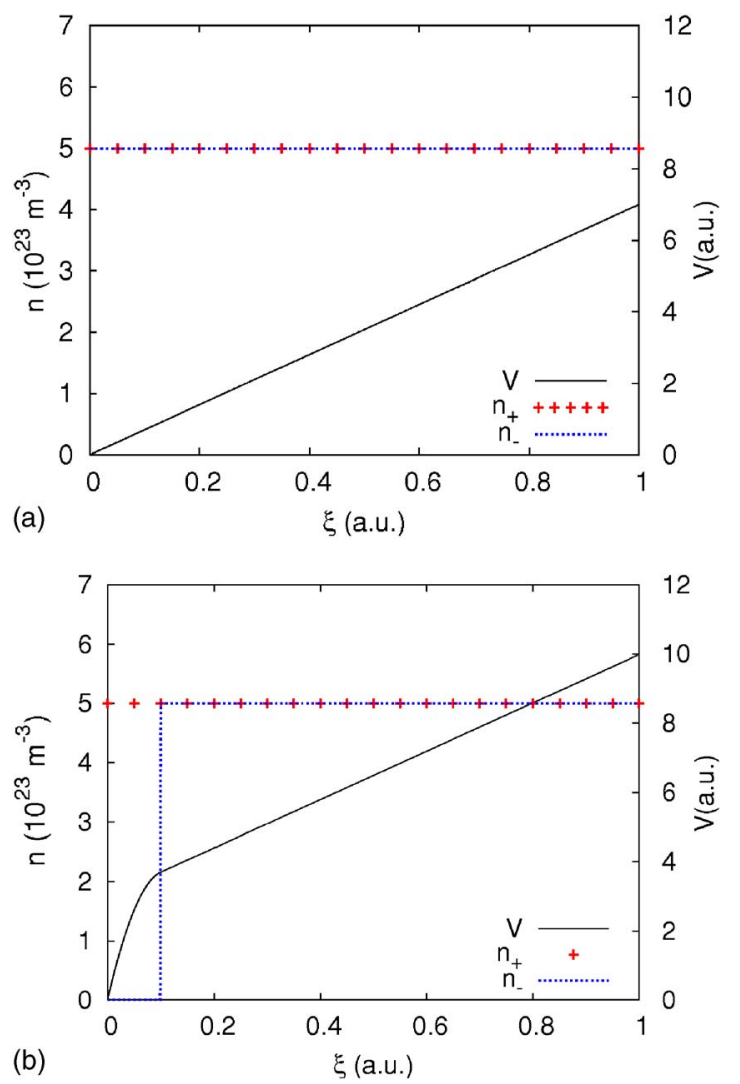

FIG. 4. (Color online) A schematic explanation of the cathode fall formation. The cathode is at $\xi=0$, and the anode is at $\xi \gg 1$. (a) The femtosecond laser pulse produces a plasma on a time scale that is essentially instantaneous for the plasma we are interested in. (b) The electrons move because of the applied electric field, creating a cathode fall in which the field is compressed. This field facilitates electron emission by the cathode. The heavier ions are essentially motionless on this time scale. 


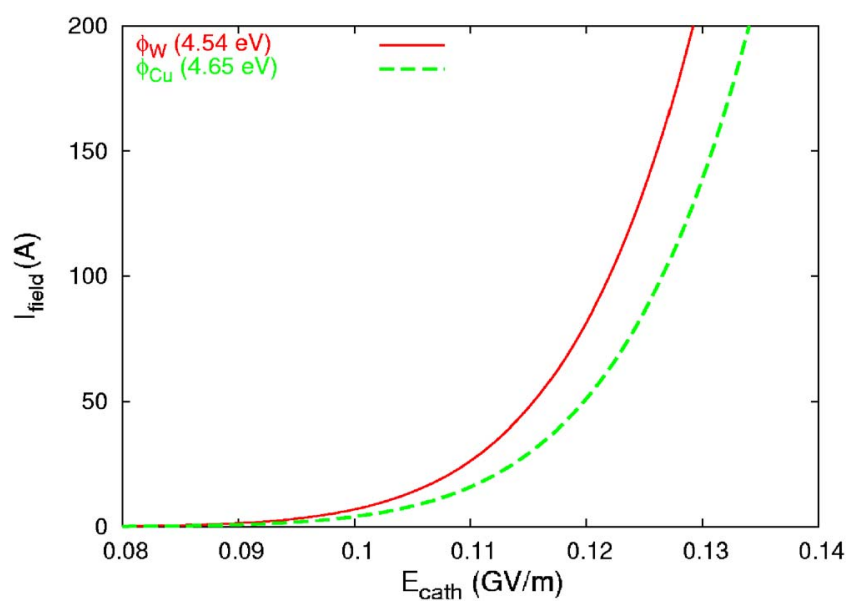

FIG. 5. (Color online) The field emission current as a function of the electric field at the cathode surface, for values of the work function corresponding to copper and tungsten cathode material. The field emission current of the actual cathode material lies between the two curves in this graph.

tributions from secondary emission might reduce the required $E_{\text {cath }}$ even further, we will use this value as an upper bound for the actual $E_{\text {cath }}$.

The cathode fall is governed by the Poisson equation

$$
\boldsymbol{\nabla} \cdot \mathbf{E}_{\mathrm{cath}}=-\nabla^{2} V_{\text {cath }}=\frac{\rho}{\epsilon},
$$

with $\rho$ the charge density and $\epsilon$ the permittivity of the plasma.

The charge density $\rho$ is determined by the initial electron density and is in our case equal to $8.0 \times 10^{4} \mathrm{C} \mathrm{m}^{-3}$. For $\epsilon$ we can take $\epsilon_{0}$, the permittivity of vacuum.

By approximating the $\boldsymbol{\nabla}$ operators with $L^{-1}, L$ being the typical length scale of the cathode fall we find that $L$ $\approx 10^{-8} \mathrm{~m}$ and $V \approx 2 \mathrm{~V}$. This cathode fall is high enough for field emission, but negligible compared to the typical voltages that are switched (a few $\mathrm{kV})$.

\section{B. The cathode fall formation time}

The formation time of the cathode fall can be estimated from the time that an electron needs to travel over a distance equal to the size of the cathode fall. This time can be calculated using

$$
q_{e} E_{\text {gap }}=m_{e} a \Rightarrow a=\frac{q V_{\text {gap }}}{m_{e} l},
$$

and

$$
L=\frac{1}{2} a t^{2} \Rightarrow t=\sqrt{\frac{2 L}{a}},
$$

where $q_{e}$ is the charge of an electron, $E_{\text {gap }}=V_{\text {gap }} / l$ is the field over the whole spark gap of length $l, a$ is the acceleration, $m_{e}$ is the electron mass, and $t$ is the time. Using Eqs. (4) and (5), the previously obtained value of $L$ and a value of $1 \mathrm{kV}$ for $V_{\text {gap }}$, we find that $t_{\text {cath }}$ is about $3 \times 10^{-13} \mathrm{~s}$. Note that this formation time is of the same order of magnitude as the inverse of the plasma frequency $f_{\mathrm{P}}$, which is equal to $6.4 \mathrm{THz}$ for the electron density under consideration (5 $\left.\times 10^{23} \mathrm{~m}^{-3}\right)$. The measurements of the switching time are limited to the measuring apparatus and were found to be shorter than $100 \mathrm{ps},{ }^{4,5}$ which is much longer than the estimated cathode fall formation time. The cathode fall formation time is also much shorter than the rise time of the electric field in the gap due to the geometry, which is a few tens of picoseconds. ${ }^{13}$ Hence, the cathode fall formation time has no influence on the rise time of the switched pulse.

\section{Conclusion}

Using an analytical model, the cathode fall formation dynamics have been investigated. The cathode fall mechanism can supply sufficient current for switching to occur. This requires a voltage which is negligible compared to the switched voltage. The cathode fall voltage rise time is much shorter than the observed rise time. Hence, the cathode fall cannot explain the observed voltage losses in the switching and does not limit the switching time. The main reason for this is the large value of $n_{e}$; for plasma with lower densities, the cathode fall may be quite significant.

\section{THE ARC PLASMA}

In this section, a numerical model of the arc that connects the electrodes will be presented. Firstly, the numerical model used will be discussed, and key assumptions are justified. Then, the model parameters, such as the current and the laser-produced plasma density which serves as a starting condition for the computation, will be presented. Based on the results from the numerical model, the general behavior of the plasma will be discussed. Finally, the impact that the finite plasma conductivity has on the switching behavior will be investigated by comparing experimental results with numerical results for various switched currents.

\section{A. The model}

The model used for the description is a two-temperature time-dependent quasineutral non-local thermal equilibrium (LTE) model. It is based on the model of a hydrogen-filled pulsed capillary discharge that is presented in Ref. 14.

For the numerical solution of the model we use the PLASIMO code. This code is described in detail in Refs. 15-19. It is a modeling platform that can handle LTE and non-LTE plasmas, currently in two dimensions. Furthermore, its modular structure allows for easy expansion of the code. It has been applied to simulate a wide variety of plasma as described in Refs. 14 and 20-26.

The system is described by a set of partial differential equations consisting of the Navier-Stokes equations that describe the bulk flow, two heat transport equations that describe the electron and heavy particle heat transport, respectively, and chemistry equations that describe the densities of individual species. For details of the equations, the reader is referred to Ref. 14.

These equations are discretized on a control volume grid that is oriented as shown in Fig. 3. The plasma is considered to be infinitely long and homogeneous in the $x$ and $y$ directions, while the computation is carried out in the $z$ direction, 
where the largest gradients are to be expected. Because it is symmetric, only the top half of the plasma is modeled.

The chemistry model assumes that the plasma is operated in pure nitrogen. The chemistry model is restricted to five species, namely, $\mathrm{N}_{2}, \mathrm{~N}, \mathrm{~N}_{2}^{+}, \mathrm{N}^{+}$, and $e$. The expected very high energy density in the system means that more complex molecular species such as $\mathrm{N}_{4}^{+}$are very rapidly destroyed and play no significant role.

Because of the high electron density $n_{e}$ of $\sim 10^{23}-10^{24} \mathrm{~m}^{-3}$, temperature $T_{e}$ of $1.5-8 \mathrm{eV}$, and relatively low heavy particle temperature $T_{h}$ of $(0.03-1.0 \mathrm{eV})$, electron kinetics dominate the reactions in the plasma. These a priori assumptions are justified by the results of the model as presented in Sec. IV D.

The following reactions are included in the model.

- Electron impact ionization of $\mathrm{N}_{2}$ described by

$\mathrm{N}_{2}+e \rightarrow \mathrm{N}_{2}^{+}+2 e$.

The rate coefficient for this equation is obtained by integrating the energy-dependent cross section with a Maxwell-Boltzmann electron energy distribution function (EEDF) for various temperatures and fitting an Arrhenius-type formula through the results. The energy-dependent cross section of this reaction is well known, and we use the value obtained by Deutsch et $a .^{27}$ from a theoretical computation, which matches earlier experimental ${ }^{28-32}$ and theoretical $^{33,34}$ results well.

The rate coefficient $k_{\mathrm{MI}}$ of this reaction as a function of $T_{e}$ is described by the Arrhenius rate

$k_{\mathrm{MI}}=3.0 \times 10^{-18}\left(T_{e}[\mathrm{~K}]\right)^{2} \exp \left(\frac{-E_{\mathrm{MI}}}{k_{B} T_{e}}\right) \mathrm{m}^{3} \mathrm{~s}^{-1}$,

with $E_{\mathrm{MI}}$ the ionization energy of $15.6 \mathrm{eV}$ and $k_{B}$ Boltz-

mann's constant. This rate describes the ionization process well (fitting error less than 6\%) between 1.5 and $10 \mathrm{eV}$. In the model, the reverse reaction is also included; its rate is computed from (7) by detailed balancing.

- Dissociative recombination of $\mathrm{N}_{2}^{+}$described by

$\mathrm{N}_{2}^{+}+e \rightarrow 2 \mathrm{~N}$.

The rate coefficient $k_{\mathrm{DR}}$ for this rapid process had been obtained by Cunningham and Hobson ${ }^{35}$ and is given by

$k_{\mathrm{DR}}=1.47 \times 10^{-12}\left(T_{e}[\mathrm{~K}]\right)^{-0.37} \mathrm{~m}^{3} \mathrm{~s}^{-1}$.

- Electron impact dissociation of $\mathrm{N}_{2}$ described by

$\mathrm{N}_{2}+e \rightarrow 2 \mathrm{~N}+e$

A recommended cross section for this process has been obtained from Cosby, ${ }^{36}$ who uses measurements and previous results by Winters. ${ }^{37}$ A good fit by an Arrhenius-type rate could only be obtained by increasing the energy threshold $E_{\text {diss }}$ from the binding energy of 9.8-11 eV. Using this fit, the following expression for the rate coefficient $k_{\text {diss }}$ is obtained:

$$
k_{\mathrm{diss}}=2.0 \times 10^{-18}\left(T_{e}[\mathrm{~K}]\right)^{0.8} \exp \left(\frac{-E_{\mathrm{diss}}}{k_{B} T_{e}}\right) \mathrm{m}^{3} \mathrm{~s}^{-1} .
$$

The fit of (11) is accurate to within $16 \%$ between 1.5 and $20 \mathrm{eV}$.

In the model, the reverse reaction is also included; its rate is computed from (11) by detailed balancing.

- Electron impact ionization of atomic $\mathrm{N}$ described by

$\mathrm{N}+e \rightarrow \mathrm{N}^{+}+2 e$

Voronov $^{38}$ gives an Arrhenius-type rate coefficient, which, with appropriate parameters, can be used to find rate coefficients for many single atoms and ions, including the rate coefficient $k_{\mathrm{AI}}$ for electron impact ionization of atomic N. $k_{\mathrm{AI}}$ is given by

$k_{\mathrm{AI}}=4.82 \times 10^{-14} \frac{1}{U+0.0652} U^{0.42} \exp (-U) \mathrm{m}^{3} \mathrm{~s}^{-1}$,

in which the reduced temperature inverse $U$ is given by

$U=\frac{E_{\mathrm{AI}}}{k_{B} T_{e}}$

According to Ref. 38, this fit is accurate to within $10 \%$ between 1 and $20 \mathrm{keV}$.

In the model, the reverse reaction is also included; its rate is computed from (14) by detailed balancing.

It is worth noting that in this plasma, the typical electron temperature is so high that direct ionization in (12) is dominant over stepwise ionization. Hence, the latter process is neglected.

Because of its crucial influence on the voltage drop over the arc, and hence on the difference between $V_{\text {in }}$ and $V_{\text {out }}$, care has been taken to accurately describe the conductivity $\sigma$ of the plasma. The description is based on the Frost mixture rule. ${ }^{39}$ In the limit of a fully singly ionized plasma, this mixture rule converges to the Spitzer-Härm value $\sigma_{\mathrm{SH}}$ of ${ }^{40-42}$

$$
\sigma_{\mathrm{SH}}=1.53 \times 10^{-2} \frac{T_{e}^{3 / 2}}{\ln \Lambda} \mathrm{Sm} \mathrm{m}^{-1},
$$

with the electron temperature $T_{e}$ in kelvins and $\ln \Lambda$ the Coulomb logarithm. For a nonfully singly ionized plasma, the collisions between electrons and neutrals, viz., $\mathrm{N}$ and $\mathrm{N}_{2}$, cause the conductivity to be lower. In order to obtain a good value for $\sigma$, it is necessary to have good values for the energy-dependent elastic momentum transfer collision cross section for both $e$ and $\mathrm{N}$, which will be denoted as $\sigma_{\mathrm{N} e}$ and for $e$ and $\mathrm{N}_{2}$, which will be denoted as $\sigma_{\mathrm{N}_{2} e}$.

A value for $\sigma_{\mathrm{N} e}$ that is valid between 0 and $1000 \mathrm{eV}$ has been obtained by combining values from Ref. 43 that give $\sigma_{\mathrm{N} e}$ for electron energies between 0 and $12 \mathrm{eV}$, and values from Ref. 44 that give $\sigma_{\mathrm{Ne}}$ for electron energies between 20 and $1000 \mathrm{eV}$. The gap in the energy range has been bridged by an interpolation. 
A value for $\sigma_{\mathrm{N}_{2} e}$ that is valid for electron energies between $10^{-2}$ and $10^{3} \mathrm{eV}$ has been obtained from Refs. 45 and 46.

For the typical $T_{e}$ in the system, these cross sections are valid for the majority of electrons. Together with the Coulomb cross section, which describes the interaction of the electron with the charged particles in the discharge, these cross sections are sufficient as input for the Frost mixture rules that describe $\sigma$.

The transport processes by the heavy particles are relatively unimportant due to the relatively high mass of the heavy particles and relatively low $T_{h}$. Their behavior will be dealt with using the default transport models in PLASIMO.

With the above mentioned model, we can simulate the transient behavior of the discharge plasma. However, the starting conditions are determined by the initial laserproduced plasma, the properties of which we will describe in the next section.

\section{B. The initial condition}

The spark gap is switched by a pulse of a femtosecondterawatt laser, which produces a plasma in a time that is much faster than all nonphotoinduced plasma processes. The composition of the initial plasma is currently not known. Hence, we will provide an estimate of the composition based on theoretical arguments.

The intense laser field of around $10^{18}-10^{19} \mathrm{~W} \mathrm{~m}^{-2}$ Ref. 4 is sufficient for photoionization to be the main ionization mechanism. ${ }^{47-49}$ This mechanism mainly produces $\mathrm{N}_{2}^{+}$, but laser-induced dissociative channels also produce $\mathrm{N}^{+}$. The higher ion states of nitrogen are neglected, as their density is lower by orders of magnitude.

Based on the measurements of the switching behavior as a function of laser intensity, ${ }^{4}$ we are lead to believe that for the laser energies used, the ionization degree is far less than unity.

The focal height of the laser has been measured, ${ }^{5}$ and based on this measurement, we will approximate the $n_{e}$ profile in this direction with a Gaussian that has a full width at half maximum of $20 \mu \mathrm{m}$. The laser-produced plasma is schematically shown in Fig. 3.

Using these estimates, we have approximated $n_{e}$ by

$$
n_{e}=5 \times 10^{23} \exp \left(\frac{-z^{2}}{2(20 \mu \mathrm{m})^{2}}\right) \mathrm{m}^{-3} .
$$

We further assume that of the corresponding ions, $90 \%$ is $\mathrm{N}_{2}^{+}$ and $10 \%$ is assumed to be $\mathrm{N}^{+} . n_{\mathrm{N}_{2}}$ is low and assumed to be $4.2 \times 10^{21} \mathrm{~m}^{-3} \cdot n_{\mathrm{N}_{2}}$ has a density that is determined such that the local mass density always matches the mass density of nitrogen at atmospheric density and room temperature.

\section{The current}

In Secs. IV A and IV B, a model for the initial conditions has been presented. The plasma evolution now depends on the current $I$ that passes through the plasma. This current is supplied by the transmission line, the amplitude depending on the charging voltage of the line, and the duration on the length of the transmission line.
When the gap is closed, the voltage is divided over the two sides of the line. This leads to a halving of the amplitude but a doubling of the duration of the current pulse of the gap. The length of the transmission line is $0.3 \mathrm{~m}$, resulting in a pulse duration of $2 \mathrm{~ns} .^{5}$

We have investigated the switching behavior for various values of the arc current. The maximum voltage across the gap that has been switched is $5 \mathrm{kV}$., ${ }^{4,5}$ For a geometrical impedance of approximately $55 \Omega,{ }^{4,5}$ this results in a blockshaped current pulse that lasts $2 \mathrm{~ns}$ and has a maximal amplitude $I_{\max }$ of $50 \mathrm{~A}$ in the ideal case, because the voltage over the gap is half the switched voltage. The spark gap plasma is simulated for various values of $I_{\max }$ between 0 and $50 \mathrm{~A}$ in increments of $5 \mathrm{~A}$.

Although the gap is closed at a femtosecond time scale by the laser pulse, the electric field that is induced by the charged transmission line needs a much longer time to adapt to this situation. In Ref. 13, it has been determined that it takes several tens of picoseconds for the electric field in the spark gap to stabilize. In the model, we approximate this by using

$$
I=\left\{\begin{array}{l}
0 \mathrm{~A}, \quad t<0 \mathrm{ps} \\
\left(\frac{t}{100 \mathrm{ps}}\right)^{0.3} I_{\max }, \quad 0 \mathrm{ps}<t<100 \mathrm{ps} \\
I_{\max }, \quad 100 \mathrm{ps}<t<2000 \mathrm{ps} \\
{\left[1-\left(\frac{t-2000 \mathrm{ps}}{100 \mathrm{ps}}\right)^{0.3}\right] I_{\max }, \quad 2000 \mathrm{ps}<t<2100 \mathrm{ps}} \\
0 \mathrm{~A}, \quad t>2100 \mathrm{ps}
\end{array}\right.
$$

to describe the instantaneous value of $I$.

The plasma evolution is most pronounced for the highest power input of $I_{\max }=50 \mathrm{~A}$. Hence, a detailed analysis of the plasma physics will be made for this case. For the other values of $I_{\max }$, the discussion will be restricted to the key quantity, the resistance.

\section{Modeling results for $I_{\max }=50 \mathrm{~A}$}

The plasma that is present in the spark gap with an $I_{\max }$ of 50 A will be discussed based on five properties: $n_{e}, T_{e}, T_{h}$, $\sigma$, and the central plasma composition.

The electron density $n_{e}$ as a function of $t$ and $z$ is presented in Fig. 6, in which two observations are made. The central $n_{e}$ rises by more than one order of magnitude during the discharge. Furthermore, the discharge does not expand much: Outside of the laser-ionized channel, there are few seed electrons, so ionization outside of the central channel is slow, and the discharge time is too short for a significant convective expansion. Between 200 ps and 2 ns, the central $n_{e}$ increases almost linearly in time. The electrons, which are heated by Ohmic dissipation, lose their heat primarily through electron impact ionization reactions. As the dissipation is roughly constant over the time range considered, the production is constant, causing the linear increase. It is worth noting that in the $2 \mathrm{~ns}$ of the discharge, no equilibrium between the Ohmic dissipation and the energy loss by thermal conduction is established, meaning there is a net ionization. 


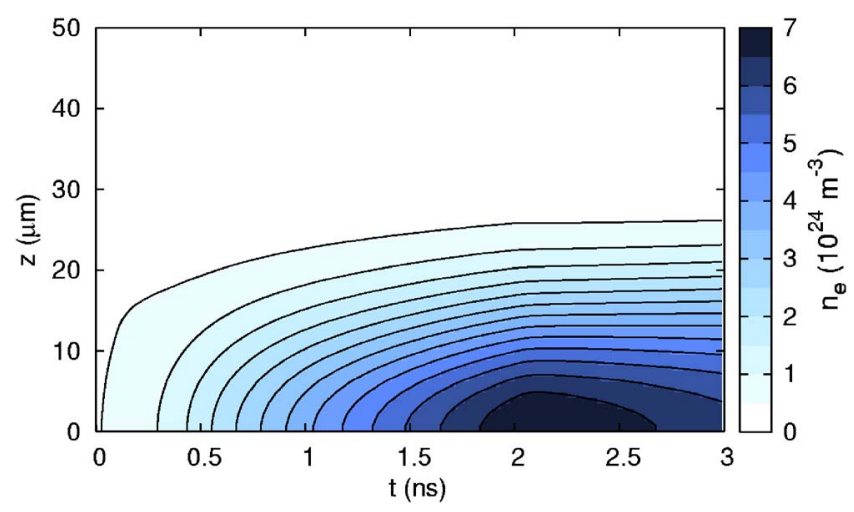

FIG. 6. (Color online) The electron density $n_{e}$ in the spark gap as a function of $t$ and $z$, for $I_{\max }=50 \mathrm{~A}$.

The electron temperature $T_{e}$ as a function of $t$ and $z$ is presented in Fig. 7. During the discharge, the plasma is coldest in the center, where the dissipation is highest. The electron density peaks in the center, causing the dissipated power per electron to be lowest there. The increase of $n_{e}$ is also causing a decrease in the dissipated power per electron and hence a decrease in $T_{e}$ in time. After the discharge, $T_{e}$ rapidly collapses, because the electrons are no longer heated by Ohmic dissipation. $T_{e}$ is then chiefly determined by the energy gained and lost due to recombination and ionization, respectively.

The heavy particle temperature $T_{h}$ as a function of $t$ and $z$ is presented in Fig. 8. In the beginning, the background gas heats up rapidly. This is due to the energy that is liberated in the dissociative recombination of $\mathrm{N}_{2}^{+}$via reaction (8). With the depletion of $\mathrm{N}_{2}^{+}$, this process becomes less important, and the heating slows down. $T_{h}$ remains lower than $T_{e}$ for the entire duration of the simulation.

$\sigma$ as a function of $t$ and $z$ is presented in Fig. 9. In the first few picoseconds, the electron temperature reaches a value that is determined by the loss from ionization and the gain from Ohmic dissipation. Because $\sigma$ depends on $T_{e}$, it also reaches a fairly stable value at this time scale. When the electron density starts increasing (cf. Fig. 6), $\sigma$ increases as well. This effect is stronger than the decrease in $\sigma$ due to the decrease in $T_{e}$. The Spitzer-Härm value $\sigma_{\mathrm{SH}}$ of Eq. (15) of

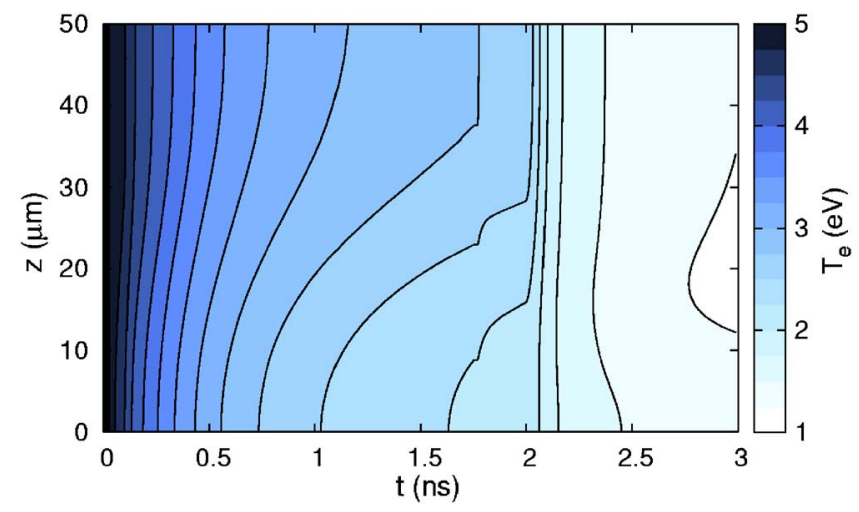

FIG. 7. (Color online) The electron temperature in the spark gap as a function of $t$ and $z$, for $I_{\max }=50 \mathrm{~A}$. The electron temperature is less relevant outside of the central ionized channel, because $n_{e}$ is very low there (cf. Fig. 6).

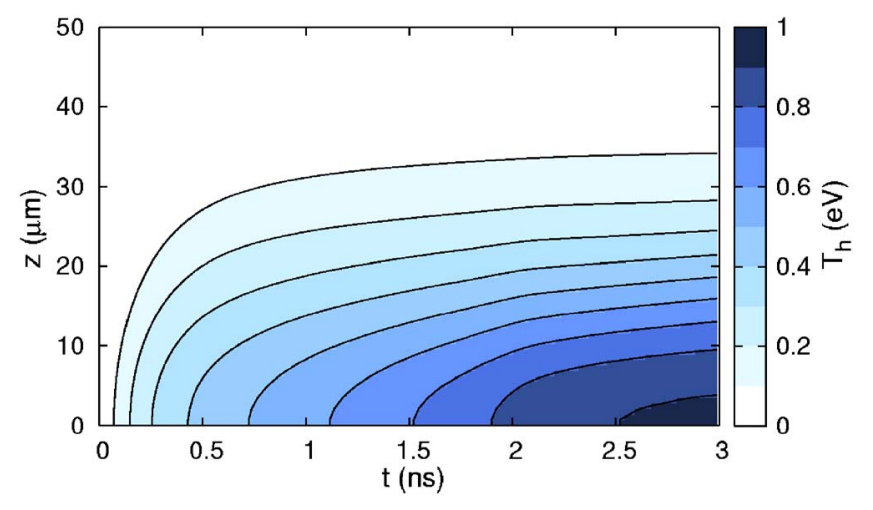

FIG. 8. (Color online) The heavy temperature $T_{h}$ in the spark gap as a function of $t$ and $z$, for $I_{\max }=50 \mathrm{~A}$.

the conductivity is not reached in this plasma. Because of the very large momentum transfer cross section between electrons and nitrogen atoms and molecules, a very high $n_{e}$ is needed to make the Coulomb collisions dominant, which is the case in the Spitzer-Härm limit.

When the current pulse ends, $T_{e}$ drops sharply, leading to a strong drop in $\sigma$. This indicates that a high $T_{e}$, and associated with this, Ohmic dissipation in the plasma, is necessary for switching.

Because the Spitzer-Härm limit has not been reached, further ionization can increase the conductivity. Because $n_{e}$ increases with increasing dissipation, as seen in Fig. 6, conductivity will be better for higher currents and hence for higher switched voltages. In other words, a smaller fraction of the voltage will be lost over the gap for higher switched voltages.

The evolution of the on-axis composition of the plasma as a function of $t$ is presented in Fig. 10. The predominant ion species in the laser-produced plasma is $\mathrm{N}_{2}^{+}$. The dissociative recombination increases for increasing $n_{e}$ and decreasing $T_{e}$ [cf. Eq. (9)]. Hence, $n_{\mathrm{N}_{2}^{+}}$drops during the evolution of the discharge. Another sharp drop is observed after the current pulse ends. At this time, $T_{e}$ drops, causing the rate of dissociative recombination to be even higher, and $n_{\mathrm{N}_{2}^{+}}$to drop further.

There is a rapid production of $\mathrm{N}$, chiefly by dissociation of $\mathrm{N}_{2}$, although dissociative recombination of $\mathrm{N}_{2}^{+}$also gives a modest contribution. This $\mathrm{N}$ is subsequently ionized to $\mathrm{N}^{+}$,

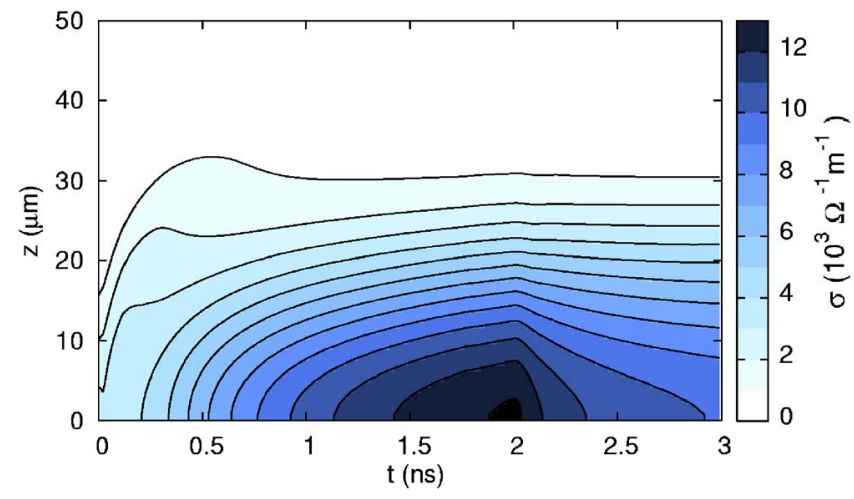

FIG. 9. (Color online) The electrical conductivity $\sigma$ in the spark gap as a function of $t$ and $z$, for $I_{\max }=50 \mathrm{~A}$. 


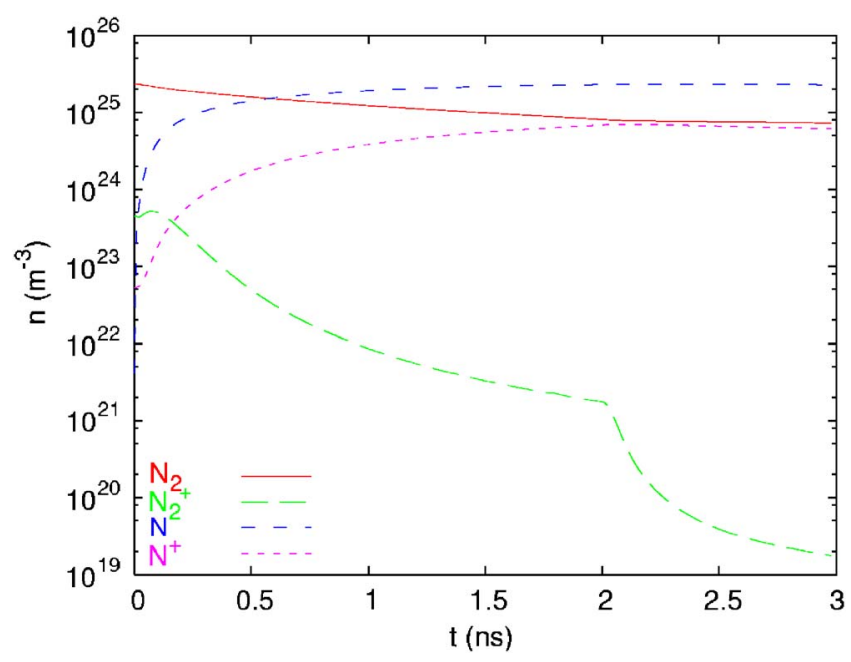

FIG. 10. (Color online) The densities of various plasma constituents as a function of $t$.

which becomes the dominant ion species. Except for $n_{\mathrm{N}_{2}^{+}}$, the plasma composition does not significantly change after the pulse ends.

\section{E. Conductivity for various currents}

One of the aims of this study is investigating the cause of the voltage that is lost in the switching. It is observed that the relative loss is larger for decreasing voltages. As mentioned in Sec. IV D, the plasma conductivity is lower for lower dissipated powers. By running the simulation for various values of $I_{\max }$, this has been verified. A typical result, the total resistance of the plasma arc $R$ at $t=2 \mathrm{~ns}$, is presented in Fig. 11.

Figure 11 shows a qualitative trend that matches the experimental observations well. The gap resistance becomes very high when little current is switched, while it is much lower, down to about $20 \Omega$, for the higher switched currents, which is much smaller than the system impedance of $55 \Omega$.

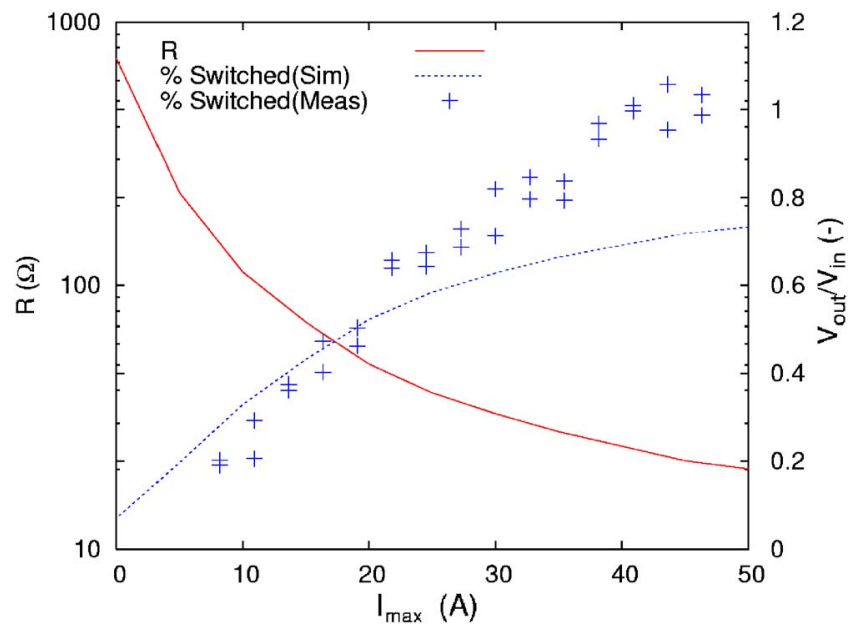

FIG. 11. (Color online) Left axis: the total resistance $R$ of the spark gap plasma at $t=2 \mathrm{~ns}$, as a function of $I_{\max }$. The resistance increases dramatically for decreasing currents. Right axis: the simulated and measured (cf. Ref. 5) percentages of the applied voltage that is switched by the spark gap. The match is good, in particular, for lower currents, although for higher currents, the simulated gap resistance is higher than the observed resistance.
For this ratio of the resistances, over $73 \%$ of the voltage on the gap is switched and $27 \%$ is dissipated. In this case, the voltage drop across the spark gap in the model is higher than in the experiment. In the low-current limit, the highest impedance of about $800 \Omega$ would mean that about $94 \%$ of the voltage is across the gap and only $6 \%$ reaches the output, meaning no switching occurs. Furthermore, the response of the resistance on the current is strongly nonlinear, with a very sharp increase in voltage drop for lower currents, as is observed in the measurements.

Quantitatively, the agreements between the simulations and the experiments are less satisfactory. The simulated resistances are a factor of 2-3 higher than the resistances that are measured. This can be explained by the poorly known starting conditions, which make a more accurate simulation of the plasma impossible. We have quantitatively analyzed the influence of the initial density. In particular, the width of the distribution was important, as the resistance of the channel is determined by the width. The exact value of the electron density is of less importance, as only a modest fraction of the electrons originates from the original plasma. Furthermore, a lower electron density leads to more dissipation and hence more electron production.

We have neglected the fact that the laser power and hence the initial plasma density are also a function of $x$, dropping off near the electrodes. This could lead to a configuration in which two zones with lower plasma density near the electrodes determine the switching behavior, while a more strongly ionized plasma in the center acts as a good conductor between these zones. Without knowledge of the initial plasma density, this hypothesis cannot be verified.

\section{CONCLUSION}

The photoconductively switched spark gap is experimentally known to be capable of switching several $\mathrm{kV}$ of voltage with jitter that is typically in the tens of picoseconds. ${ }^{4,5}$ Voltages down to $10 \%$ of the self-breakdown voltage can be switched. One presently unresolved question is the cause of the voltage drop over the gap, which is most pronounced for lower voltages. We have investigated two plausible causes of this behavior: the cathode fall and the finite resistance of the arc plasma in the spark gap.

Based on a simple, analytical model of the cathode fall, the formation time is estimated to be less than $300 \mathrm{fs}$, while the voltage drop over the cathode fall is less than $2 \mathrm{~V}$. The formation time is orders of magnitude shorter than the geometric rise time of the switched pulse and is therefore not limiting in the switching behavior. The cathode voltage drop is typically one-tenth of a percent of the switched voltage and is much smaller than the experimentally observed voltage drops across the spark gap. From this, we conclude that the cathode fall behavior cannot be the cause of the observed voltage drop.

A non-LTE model has been used to compute the plasma properties of the arc plasma, including the electrical resistance of the spark gap plasma that bridges the electrodes. Due to the poorly known initial conditions, the model cannot be expected to give a full quantitative explanation of the 
switching behavior of the spark gap. Qualitatively, the arc plasma becomes a good electrical conductor for higher switched voltages, with a maximum switched voltage of $70 \%$ for the current range investigated, and a poor conductor for lower switched voltages, switching $6 \%$ in the limit of no voltage. The strong nonlinearity of the switching behavior, i.e. a strong decrease in the switching efficiency for lower voltages, as is experimentally observed is also present in the modeled results. Based on this, we conclude that the voltage drop across the plasma is likely caused by the finite resistance of the arc plasma.

Quantitatively, the match is very good for the lower current, but somewhat less so for the higher currents, as experimentally, virtually all the voltage is switched for higher voltages, while in the model, a maximum of $73 \%$ of the voltage is switched.

The successful modeling of the plasma proves the feasibility of modeling the plasma in a photoconductively switched spark gap. The PLASIMO code is capable of simulating the complex, strongly dynamic behavior of the plasma on these short time scales.

\section{ACKNOWLEDGMENTS}

The PLASIMO team members, current and former, are acknowledged for their contributions to the code. This work was funded by the Technology Foundation STW, Applied Science Division of NWO and the technology program of the Ministry of Economic Affairs, the Royal Netherlands Academy of Arts and Sciences, and the Foundation for Fundamental Research on Matter (FOM).

${ }^{1}$ S. B. van der Geer, M. J. de Loos, G. J. H. Brussaard, O. J. Luiten, and M. J. van der Wiel, in Proceedings of the Seventh European Particle Physics Conference, Paris, France, 2002 (unpublished), p. 989.

${ }^{2}$ M. J. Kushner, R. D. Milroy, and W. D. Kimura, J. Appl. Phys. 58, 2988 (1985).

${ }^{3}$ A. H. Guenther and J. R. Bettis, J. Phys. D 11, 1577 (1978).

${ }^{4}$ G. J. H. Brussaard and J. Hendriks, Appl. Phys. Lett. 86, 081503 (2005).

${ }^{5}$ J. Hendriks, B. H. P. Broks, J. J. A. M. van der Mullen, and G. J. H. Brussaard, J. Appl. Phys. 98, 043309 (2005).

${ }^{6}$ R. H. Fowler and L. Nordheim, Proc. R. Soc. London, Ser. A 119, 173 (1928).

${ }^{7}$ High-Voltage Technology, edited by L. L. Alston (Oxford University Press, London, 1968).

${ }^{8}$ Handbook of Accelerator Physics and Engineering, edited by A. W. Chao and M. Tigner (World Scientific, Singapore, 1998).

${ }^{9}$ B. J. Hopkins and J. C. Riviere, Proc. Phys. Soc. London 81, 590 (1963).

${ }^{10}$ P. O. Gartland, S. Berge, and B. J. Slagsvold, Phys. Rev. Lett. 28, 738 (1972).
${ }^{11}$ M. D. Lang and W. Kohn, Phys. Rev. B 3, 1215 (1971).

${ }^{12}$ S. C. Fain Jr. and J. M. McDavid, Phys. Rev. B 9, 5099 (1974).

${ }^{13}$ J. Hendriks, S. B. van der Geer, and G. J. H. Brussaard, J. Phys. D 38, 2798 (2005).

${ }^{14}$ B. H. P. Broks, K. Garloff, and J. J. A. M. van der Mullen, Phys. Rev. E 71, 016401 (2005).

${ }^{15}$ J. van Dijk, Ph.D. thesis, Eindhoven University of Technology, 2001.

${ }^{16}$ G. M. Janssen, Ph.D. thesis, Eindhoven University of Technology, 2000.

${ }^{17}$ H. van der Heijden, Ph.D. thesis, Eindhoven University of Technology, 2002.

${ }^{18}$ A. Hartgers, Ph.D. thesis, Eindhoven University of Technology, 2003.

${ }^{19}$ http://plasimo.phys.tue.nl

${ }^{20}$ K. T. A. L. Burm, W. J. Goedheer, J. A. M. van der Mullen, G. M. Janssen, and D. C. Schram, Plasma Sources Sci. Technol. 7, 395 (1998).

${ }^{21}$ G. M. Janssen, J. van Dijk, D. A. Benoy, M. A. Tas, K. T. A. L. Burm, W. J. Goedheer, J. A. M. van der Mullen, and D. C. Schram, Plasma Sources Sci. Technol. 8, 1 (1999).

${ }^{22}$ H. van der Heijden and J. van der Mullen, J. Phys. B 34, 4183 (2001).

${ }^{23} \mathrm{H}$. van der Heijden, J. Baier, and J. van der Mullen, J. Phys. B 35, 3633 (2002).

${ }^{24}$ H. van der Heijden and J. van der Mullen, J. Phys. D 35, 2112 (2002).

${ }^{25}$ J. van Dijk, M. van der Velden, and J. van der Mullen, J. Phys. D 35, 2748 (2002).

${ }^{26}$ A. Hartgers, H. W. P. van der Heijden, M. L. Beks, J. van Dijk, and J. A. M. van der Mullen, J. Phys. D 38, 3422 (2005).

${ }^{27}$ H. Deutch, K. Becker, S. Matt, and T. D. Märk, Int. J. Mass. Spectrom. 197, 37 (2000).

${ }^{28}$ D. Rapp and P. Englander-Golden, J. Chem. Phys. 43, 1465 (1965).

${ }^{29}$ B. L. Schram, F. J. de Heer, M. J. van der Wiel, and J. Kistemaker, Physica 35, 94 (1965).

${ }^{30}$ B. L. Schram, H. R. Moustafa, J. Schutten, and F. J. de Heer, Physica 36, 734 (1966).

${ }^{31}$ E. Krishnakumar and S. K. Srivastava, J. Phys. B 23, 1893 (1990).

${ }^{32}$ H. C. Straub, P. Renault, B. G. Lindsay, K. A. Smith, and R. F. Stebbings, Phys. Rev. A 54, 2146 (1996).

${ }^{33}$ W. Hwang, Y.-K. Kim, and M. E. Rudd, J. Chem. Phys. 104, 2956 (1996).

${ }^{34}$ V. Saksena, M. S. Kushwaha, and S. P. Khare, Physica B 233, 201 (1997).

${ }^{35}$ A. J. Cunningham and R. M. Hobson, J. Phys. B 5, 2328 (1972).

${ }^{36}$ P. C. Cosby, Chem. Phys. 98, 9544 (1993).

${ }^{37}$ H. F. Winters, J. Chem. Phys. 44, 1472 (1966).

${ }^{38}$ G. S. Voronov, At. Data Nucl. Data Tables 65, 1 (1997)

${ }^{39}$ L. S. Frost, J. Appl. Phys. 32, 2029 (1961)

${ }^{40}$ R. S. Cohen, L. Spitzer, Jr., and P. McR. Routly, Phys. Rev. 80, 230 (1950).

${ }^{41}$ L. Spitzer, Jr. and R. Härm, Phys. Rev. 89, 977 (1953).

${ }^{42}$ M. Mitchner and C. H. Kruger, Partially Ionized Gases (Wiley, New York, 1983).

${ }^{43}$ K. A. Berrington, P. G. Burke, and W. D. Robb, J. Phys. B 8, 2500 (1975).

${ }^{44}$ T. Wedde and T. G. Strand, J. Phys. B 7, 1091 (1974).

${ }^{45}$ A. Okhrimovskyy, A. Bogaerts, and R. Gijbels, Phys. Rev. E 65, 037402 (2002).

${ }^{46}$ A. Phelps and L. C. Pitchford, Phys. Rev. A 31, 2932 (1985).

${ }^{47}$ C. Guo and G. N. Gibson, Phys. Rev. A 63, 040701(R) (2001).

${ }^{48}$ J. P. Nibarger, S. P. Menon, and G. N. Gibson, Phys. Rev. A 63, 053406 (2001).

${ }^{49}$ L. V. Litvinyuk, K. F. Lee, P. W. Dooley, D. M. Rayner, D. M. Villeneuve, and P. B. Corkum, Phys. Rev. Lett. 90, 233003 (2003). 